\title{
Las ciudades de Castilla-La Man- cha en el viaje de Andrea Navagero
}

\author{
VERÓNICA GIJÓN JIMÉNEZ \\ Profesora Ayudante. Departamento de Historia del Arte \\ Universidad de Castilla-La Mancha \\ veronica.gijon@uclm.es
}

\begin{abstract}
Resumen
El veneciano Andrea Navagero visitó Castilla-La Mancha entre 1525 y 1526, para cumplir su misión como embajador ante Carlos $\mathrm{V}$. Al contrario que otros embajadores, que no solían darle mucha importancia a la descripción de las ciudades, Navagero nos dejó interesantes descripciones. El autor se centra, sobre todo, en las ciudades más importantes, Toledo y Guadalajara. La primera de ellas era sede temporal de la Corte de Carlos V y vivía un momento de esplendor. Guadalajara alojaba a la poderosa familia Mendoza, que de facto, eran los señores de la ciudad. Navagero se centra sobre todo en la descripción del urbanismo, de los edificios más emblemáticos y en algunos aspectos sociales. Gracias a su vasta cultura y a sus grandes dotes de observador, el embajador veneciano nos muestra un completo panorama de estas dos ciudades en uno de los momentos más importantes de su historia.
\end{abstract}

Palabras clave: Castilla-La Mancha, ciudad, literatura de viajes, Navagero, patrimonio artístico.

\section{The cities of Castilla-La Mancha in the journey of Andrea Navagero}

\begin{abstract}
The venetian Andrea Navagero visited Castilla-La Mancha between 1525 and 1526, in order to carry out his mission as ambassador before Charles V. Unlike other ambassadors, that didn't use to attach importance to the description of cities, Navagero provided us with interesting descriptions. The author especially focuses on more important cities, Toledo and Guadalajara. The first of them was provisional seat of the Charles V's court and experienced a time of magnificence. Guadalajara housed the powerful family Mendoza, who were in fact, the gentlemen of the city. Navagero focuses, above all, on the description of town planning, the more emblematic building and finally on some social aspects. Thanks to his vast culture and his observer qualities, the Venetian ambassador shows us a complete scene of both cities in one of the most important moments in history.
\end{abstract}


Key words: Artistic heritage, Castilla-La Mancha, city, Navagero, travel literature.

Sumario: 1.- Introducción. 2.- Guadalajara y sus palacios. 3.- La Corte de Carlos V en Toledo. 4.- El arte y las ciudades en el relato de Navagero. 5.- Bibliografía.

\section{Introducción.}

Una parte importante de los viajeros que atravesaron Castilla-La Mancha durante la Edad Moderna eran embajadores. Entre ellos destacan los enviados por la República de Venecia, uno de los primeros Estados en establecer agentes permanentes en todas las cortes. Además los embajadores venecianos estaban obligados desde 1268 a leer ante el senado una relación de su embajada a los quince días de su regreso. Estos relatos servían para trasmitir sus conocimientos al gobierno y a la oligarquía dirigente. A partir de 1425 estos relatos comenzaron a ser archivados en la cancillería secreta (Rivero Rodríguez, 2000: 28-35).

Gracias a las relaciones de las embajadas, tenemos unos documentos muy valiosos para el estudio de la historia de España. Estos autores se solían centrar en temas políticos o militares y muy pocas veces reparaban en las descripciones de las ciudades y sus edificios más representativos. Esto se debía a que no lo consideraban una información útil para los gobiernos de sus países. Sin embargo, algunos embajadores sí le dieron importancia a estos asuntos; y las razones para hacerlo las expuso en su relación de embajada uno de los enviados de la República de Venecia, Tommaso Contarini, que afirmaba que el conocimiento de los estados y provincias que pertenecían a un príncipe era importante para saber cuánto había que apreciarlos o temerlos (Contarini, 1666: 1-2). Dentro del grupo de los embajadores venecianos Andrea Navagero ${ }^{1}$ es una excepción, porque nos dejó un largo relato de su viaje a España entre 1524 y 1526. En este caso, los ojos que contemplan nuestro país son los de un huma-

\footnotetext{
${ }^{1}$ Andrea Navagero prestaba más atención al arte que otros embajadores. Lo mejor descrito en su relato son los paisajes y los jardines, por sus preferencias personales. Cuando visitaba alguna ciudad, solía dar cuenta de los edificios más importantes, aunque sus descripciones no eran muy prolijas. Toledo es una de las ciudades sobre las que más escribió en el conjunto del relato. También suele hacer una descripción general de las ciudades.
} 
nista proveniente de Italia, que tuvo un importante papel en la introducción de los nuevos metros renacentistas en nuestra poesía. Fue él quien sugirió a Boscán que escribiera versos en castellano siguiendo las novedades que se habían dado en su país (Colombi Monguió, 1992: 143-168).

Para entender el relato de Navagero debemos reparar en su peculiar personalidad, que impregna la totalidad de su texto. Estamos ante un hombre de gran cultura con grandes dotes de observador, pero que, sin embargo, cuando habla de los edificios no hace descripciones demasiado extensas. Sólo se emociona ante los jardines que va encontrando a lo largo de su viaje, que eran su gran afición. Él mismo poseía dos, que había dejado al cuidado de sus amigos, a los que escribe a lo largo de todo su viaje refiriéndoles los detalles de su recorrido y preocupándose por el estado de sus jardines. Tenía una mentalidad muy abierta para su época y no tenía prejuicios para alabar o criticar lo que encontraba a su paso (Lupi, 1997: 529-532). Muestra de ello es su visita a Granada, en la que elogia a los moriscos por sus bonitos jardines y su laboriosidad y critica la decisión de instaurar la Inquisición en la ciudad y la pereza de los españoles, que terminarán por arruinarla. En cambio Navagero critica la construcción de nuevas iglesias en la ciudad, ya que habría preferido que perduraran los jardines musulmanes (Navagero, 1983: 45-61). El embajador embarcó en Génova en 1525 y llegó a Palamós el 24 de abril del mismo año, e inmediatamente se dirigió a Toledo, donde estaba la Corte. Antes pasó por Guadalajara procedente de Zaragoza, mencionando los lugares que encontraba en su recorrido: Sigüenza, Trijueque, Jadraque, Padilla e Hita, pero no describió nada de ellos.

\section{Guadalajara y sus palacios.}

La primera ciudad que describe con más detenimiento es Guadalajara. Destaca la calidad de sus casas, sobre todo, se fija en el palacio del Cardenal Mendoza y el del Duque del Infantado, al cual considera el más bello de España. El primer edificio al que se refiere el autor estaba situado frente a la iglesia de Santa María y no ha llegado hasta nuestros días porque fue destruido por un incendio en el siglo XVIII (Fernández Madrid, 1991: 253). En origen era una residencia medieval urbana heredada de su padre el Marqués de Santillana. Posteriormente habría sido reformada con trazas de Lorenzo Vázquez para adaptarlo a los nuevos gustos renacentistas. El edificio debía estar terminado hacia 1491, según una lápida que se colocó en el zaguán (Romero Medina, 2010: 1-3). Otro viajero que 
visitó Guadalajara unos años antes que Navagero, nos dejó una descripción más detallada, que nos permite hacernos una idea de cómo podía ser este edificio:

La casa del Cardenal, en las afueras de Guadalajara, es la más bella de toda España. Yo he visto muchos grandes palacios de cardenales en Roma, pero en toda mi vida jamás vi otro tan cómodo y con las habitaciones tan bien distribuidas. Tiene dos bellísimos claustros superpuestos, con pequeñas salas y cámaras, todas con artesonados dorados y diversos colores mezclados con azul, siendo cada artesonado diferente de los otros; dos salas de verano abiertas al jardín con columnas de mármol y refulgentes de tanto oro, que es difícil de creerse. ¡Oh qué majestuosa capilla! Larga, aunque no muy ancha, en cuyo altar hay exquisitas pinturas de San Pedro y San Pablo y de la bienaventurada Virgen, y a los lados San Gregorio y Santa Elena con la cruz... (Münzer, 1991: 283).

El otro edificio al que se refiere Navagero es el Palacio del Infantado, construido por orden del segundo duque del Infantado, don Iñigo López de Mendoza, sobre el solar de la antigua casa de la familia, que fue derribada. El edificio estaba terminado en lo esencial en 1492, como reza la inscripción que se encuentra en el salón de Linajes. El artífice de la obra fue Juan Guas, ayudado por otro maestro de difícil identificación, que podría ser Enrique Egas. También participaron Lorenzo de Trillo y otros artistas de Guadalajara y de pueblos vecinos, algunos de ellos moriscos. El resultado es un edificio gótico flamígero, con una fuerte influencia del mudéjar y un leve influjo renacentista (Layna, 1997: 19-29).

Navagero es el primer viajero que dice que la ciudad es del rey, aunque el duque puede considerarse en la práctica como el señor del lugar. A pesar de que sus rentas eran muy altas, cincuenta mil ducados, no llegaban para cubrir sus gastos, ya que era muy dado al dispendio. Navagero nos dice que el duque tenía doscientos peones, hombres de armas y una capilla de músicos, además se mostraba muy liberal en todo (Navagero, 1983: 23-24). Navagero tenía mucha razón en esto, pues era bien conocida la inclinación de los Mendoza al dispendio, sobre todo el segundo duque, que era muy dado al lujo y la ostentación en todo lo que emprendía, ya fuera una campaña militar como la de Granada, la recepción de algún invitado ilustre o la construcción del palacio del Infantado (Layna, 1994: 226). Navagero salió de Guadalajara pasando por un puente de piedra con una torre en el centro. Se trata de un puente construido por los musulmanes y reformado por Alfonso X (Sánchez Doncel, 1984: 235-236). 


\section{La Corte de Carlos V en Toledo.}

Después fue a Alcalá de Henares y a Madrid, dirigiéndose luego a Toledo. Allí llegó el 11 de junio de 1525. En primer lugar describe el emplazamiento de la ciudad haciendo referencia al clima y a la vegetación, especialmente a la Huerta del Rey y la Vega. Después pasa a ocuparse de las ruinas romanas: un acueducto y el circo, que aún hoy podemos ver. Del acueducto nos quedan pocos restos, por los que sabemos que estaría situado cerca del puente de Alcántara. Esta construcción abastecía de agua a la ciudad de Toledo transportándola desde la sierra de los Yébenes (Moreno, 2002: 159). El circo romano estaría muy dañado en esta época, porque sus materiales se habían aprovechado para otros edificios (SánchezPalencia, 1988: 5-8). Habla de la estrechez de sus calles y de la ausencia de espacios abiertos dentro de la ciudad salvo la plaza de Zocodover, que todavía no había sufrido el terrible incendio que la destruyó a finales de ese mismo siglo.

Fig. 1. Puente de San Martín, Toledo, principios del siglo XIII. Foto V. Gijón.

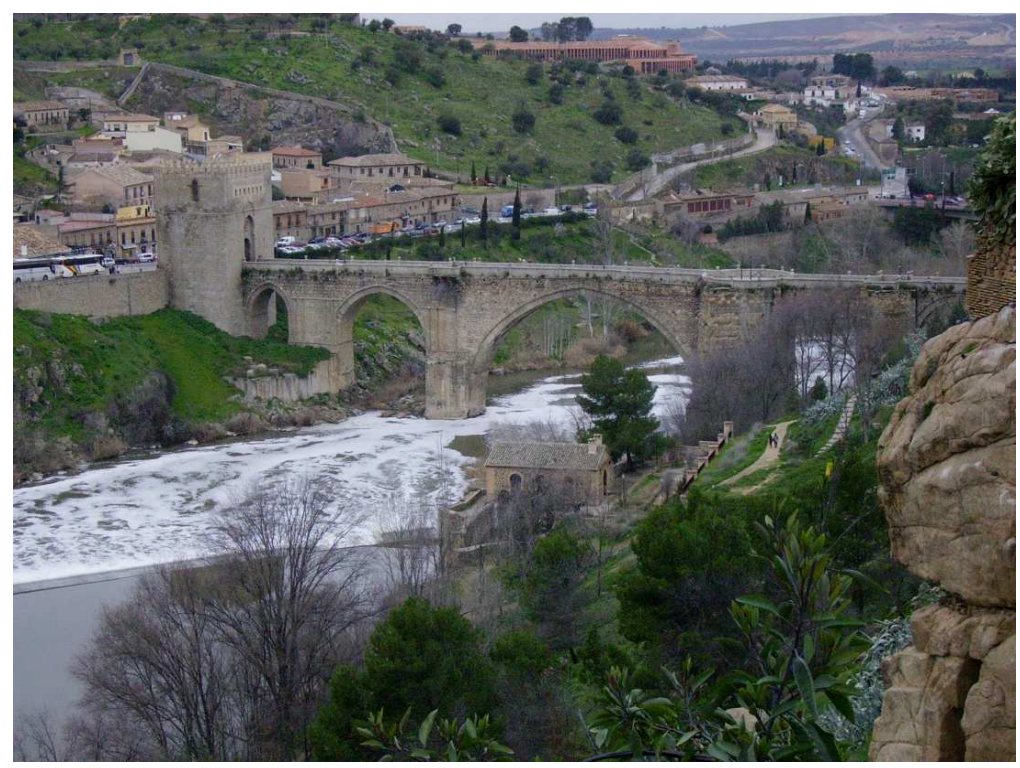

También hace referencia a las salidas y entradas de la capital, las puertas del Cambrón y de Bisagra y los puentes de Alcántara y San Martín. La puerta de Bisagra aún no había sufrido la reconstrucción de 1545 (Marías, 1985: 50-53), ni la puerta del Cambrón la remodelación de 1571; de manera que Navagero vio las puertas musulmanas, pero no las describe. El puente de Alcántara, de construcción romana, aún no tenía su arco de entrada, edificado en 1721 para sustituir un 
torreón árabe. El puente de San Martín se construyó después de la riada de 1203, que destruyó el puente de barcas que había cerca del torreón de la Cava (Carrero de Dios, 1981: $32-37$ y 52-53). También nos habla Navagero de un artilugio diseñado para sacar agua del Tajo, que en ese momento no funcionaba, y se había encargado a un ingeniero que lo activase. Este artilugio no era el de Juanelo Turriano, puesto que éste se construyó a partir de 1565. Navagero debe referirse a uno de los intentos que se realizaron para subir agua del Tajo antes de que llegara a España el relojero cremonés. Navagero hablaba de los oficiales que se enviaron desde Alemania en 1526 (Navagero, 1983: 110), que instalaron un sistema de bombas y tuberías, que no dieron el resultado esperado, puesto que la presión del agua lo reventó (Porres, 1987: 19-20).

Después se dirigió a uno de los edificios más frecuentados por los viajeros, la Catedral. Lo que más le sorprendió de ella, como al resto de los viajeros de su tiempo, fue el tesoro, especialmente la custodia, que estaba recién acabada. Enrique de Arfe había terminado hacía poco el tabernáculo que rodeaba a la custodia, encargado por Isabel de Castilla al orfebre Almarique. Esta pieza había sido comprada por orden del cardenal Cisneros en la testamentaría de la reina en Toro, el 13 de marzo de 1505 (Zalama, 2005: 331-353). Años más tarde, se pensó que la Catedral necesitaba una custodia monumental para ensalzar la fiesta del Corpus Christi. Por este motivo, se decidió construir un tabernáculo que enmarcara a la custodia de la reina según el proyecto de Copín de Holanda, que copiaba el tabernáculo del retablo de la Catedral. Para la realización del proyecto se llamó al mejor orfebre del momento, Enrique de Arfe, que había venido de Flandes con Felipe el Hermoso. El artífice tardó nueve años en finalizar la custodia que hoy conocemos, ya que la comenzó en el 23 de octubre del 1515 y finalizó el 23 de abril de 1524 (Arteaga, 1924: 238254). Navagero la contempló un año y dos meses después de esa fecha. Estaba elaborada en estilo gótico, y el viajero quedó sorprendido ante su riqueza.

Cuando él la vio ya debía de haberse realizado la reforma ordenada por el arzobispo Fonseca, que quiso que Enrique de Arfe sustituyera todas la piezas de hierro que tenía la custodia por otras de plata, fundamentalmente el armazón y la base, de manera que la pieza ya sólo estuvo compuesta por oro, plata y piedras preciosas, y así es como la describe nuestro viajero (Fernández Collado, 1999: 147-152). En la carta que escribió a su amigo Ramusio el doce de septiembre de 1525 es más explícito en su descripción: Entre otras cosas hay 
una Custodia o tabernáculo para poner el cuerpo de Nuestro Señor Jesucristo, de oro y plata con piedras preciosas, que dicen vale treinta mil ducados, y en verdad es bellísima y soberbia (Navagero, 1983: 112).

Otro aspecto importante en el que repara Navagero es en la riqueza del clero toledano; dice que son los dueños de la ciudad y tienen las mejores casas, ya que era muy numeroso y tenía elevadas rentas. Antes de visitar la Catedral recorre el palacio Arzobispal que es harto bueno (Navagero, 1983: 27). Es una lástima que Navagero no lo describiera, puesto que es uno de los edificios toledanos que más ha cambiado a lo largo de la historia. Su origen está en unas casas que Alfonso VIII donó al arzobispo Rodrigo Jiménez de Rada para que fuese su vivienda. A partir de aquí, los siguientes arzobispos continuaron haciendo reformas hasta componer el palacio actual. Navagero debió de ver el pasadizo que había hecho construir el arzobispo Mendoza para comunicar el palacio con la Catedral (Peris, 1991: 387).

La nobleza también era un estamento importante, y así lo refleja el autor en su relato. Habla de los principales nobles de la ciudad como los jefes de sus principales casas nobiliarias, el conde de Fuensalida de la Casa de Ayala y don Juan de Rivera de la casa de Silva, que tenían una gran rivalidad entre sí. De la nobleza dice que aparentan más riqueza de la que en realidad tienen, y que algunos poseen bellos palacios en la ciudad. No habla de los palacios en el relato de su viaje, pero podemos encontrar más datos en la carta a Ramusio, donde describe el típico palacio toledano.

Matiza que por fuera no tienen mucha apariencia, están hechos de cantos y de piedras por algunas partes y no tienen casi ventanas ni balcones debido, según el autor, al calor y el frío. Se articulan en torno a un patio con cuatro crujías que se construye en el centro (Navagero, 1983: 113). No se le ocurre a Navagero que la tradición musulmana pesaba todavía sobre la arquitectura civil (Marías, 1983: 166), pero también es cierto que aún no había estado en Granada ni en Sevilla. A pesar de que dice que estos palacios son buenos y cómodos debieron resultarle poco llamativos en comparación con los italianos, sobre todo por la pobreza de sus materiales.

Finalmente Navagero hace una pequeña referencia al Hospital de Santa Cruz. Nos habla de su localización cerca de la puerta de Alcántara y dice que es hermosísimo, muy bien labrado y que nada le falta (Navagero, 1983: 29). 
Cuando Navagero visitó este hospital debía estar prácticamente acabado. No advierte la influencia del Renacimiento italiano en la planta, ni tampoco en los motivos decorativos. Es cierto que también se utilizan algunas soluciones góticas, y no falta tampoco la influencia del mudejarismo en las armaduras de madera que cubren las naves (Santolaya, 1990: 317-318). Su fundación había sido estipulada por el cardenal Mendoza en su testamento, pero no se comenzó a construir hasta 1504 bajo el atento seguimiento de los albaceas testamentarios del cardenal. Entre ellos la reina Isabel, que también financió la construcción de los hospitales de Granada y Santiago. Las obras del hospital estaban muy avanzadas, pero Navagero no debió ver el patio y las escaleras de Covarrubias, puesto que los documentos existentes hacen pensar que ambos fueron construidos en torno a $1535^{2}$.

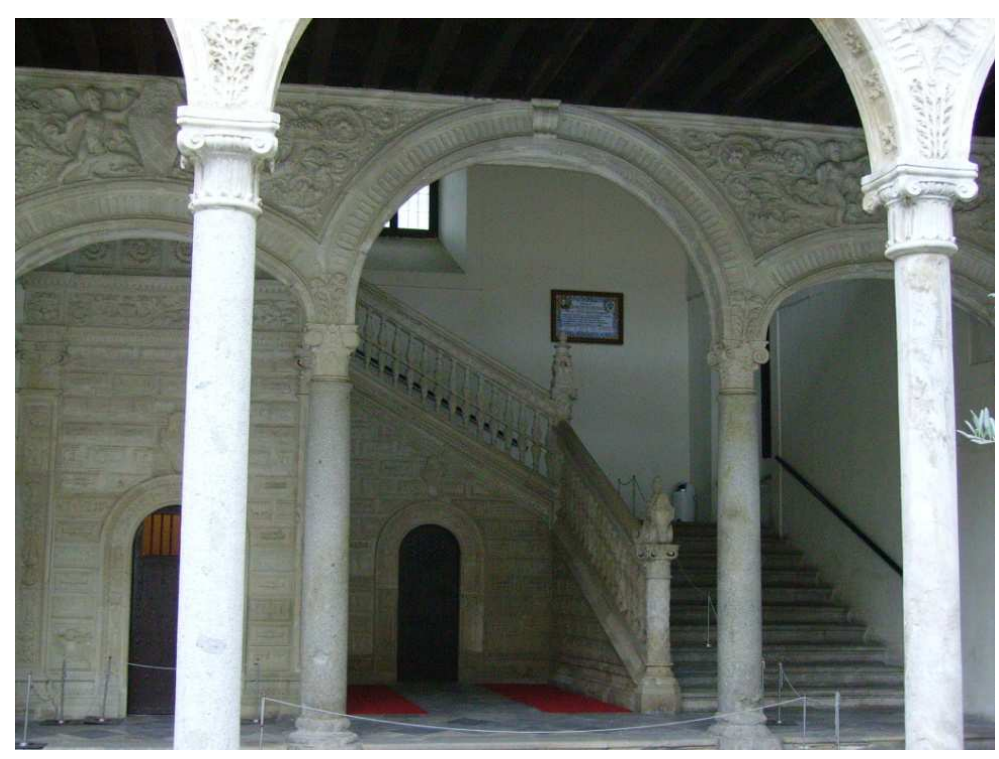

Fig. 2. Escalera plateresca del hospital de Santa Cruz de Toledo, c. 1535. Foto $V$. Gijón.

\footnotetext{
${ }^{2}$ Un documento fechado el 2 de mayo de 1535 recoge las condiciones del cantero Juan de Plasencia para reparar el patio principal. Alonso de Covarrubias aparece como su fiador. El patio descrito estaba en muy malas condiciones, y tenía elementos como gárgolas o arbotantes, que nos hacen pensar que era de estilo gótico. Este patio podría haber sido construido en la fase de obras anterior a 1514, fecha de la inauguración del hospital. No sabemos por qué se construyó un patio nuevo en lugar de arreglar el anterior, pero la escalera y el patio datarían de la fecha que indica este documento (Diez del Corral, 1984: 161-182).
} 
Navagero estuvo con la Corte en Toledo más de ocho meses, hasta que en febrero de 1526, el rey decidió ir a Sevilla. Para llegar a Andalucía siguió el itinerario que atravesaba Extremadura, pasando por Talavera de la Reina, un buen lugar situado a orillas del Tajo, sobre el cual hay un puente y donde se conservan restos de una muralla antigua, una puerta y ruinas de termas con inscripciones donde se lee el nombre de Gneo Pompeyo (González Moreno, 1999-2000: 226).

La muralla a la que se refiere son los restos de lienzos romanos utilizados por los musulmanes en la construcción de su propia muralla. La puerta de la que habla debe ser la de San Pedro, ensanchada por el arzobispo Mendoza en 1494. La inscripción que menciona Navagero también fue registrada por el padre Mariana y decía Gneo Pompeyo hijo del gran Pompeyo (Ballesteros, 1981: 46). Talavera fue la última ciudad que describe antes de salir de Castilla-La Mancha por Puente del Arzobispo (Navagero, 1983: 30-31).

Cuando la Corte regresó desde Sevilla a Madrid, lo hicieron atravesando las tierras de La Mancha. Cruzaron Sierra Morena llegando a Viso del Marqués y no menciona el palacio de Don Álvaro de Bazán. Desde El Viso fueron a Almagro, sede de los maestres de la orden de Calatrava; no destaca ninguno de sus edificios, sólo indica que en la ciudad hay varios pozos de agua agria. En este punto alude a Almadén y sus minas de mercurio. Antes de salir definitivamente de Almagro visitaron Carrión y Malagón, dejando a su derecha Calatrava la Vieja, situada en un cerro, rodeada de riscos, pero desierta y arruinada por la insalubridad causada por el río que allí es pantanoso. Al salir de Almagro definitivamente dejaron a Ciudad Real a la derecha y llegaron a Yébenes. Antes vieron los restos de un antiguo acueducto que llevaba agua hasta Consuegra. Posiblemente se refiera a los restos del acueducto de Toledo, que como ya hemos visto comenzaba en los Yébenes. Finalmente llegaron a Madrid pasando por Orgaz y Toledo (Navagero, 1983: 68-69).

Calatrava la Vieja habría sido abandonada por la Orden hacia 1217, pero su población permaneció allí. A principios del siglo XVI tenía 200 vecinos, pero en las relaciones topográficas de 1575 ya estaba totalmente despoblada (Ruibal, 1984: 70-71). Según el testimonio de Navagero estaría despoblada en 1525. Es comprensible que no hable sobre El Viso porque en esta época aun no existía el palacio del marqués de Santa Cruz (Corchado, 1982: 65-66). Sin embargo, es 
sorprendente que no reseñe ninguno de los edificios de Almagro que, como bien dice Navagero, era cabeza de la orden de Calatrava y lugar de residencia de los banqueros alemanes Fúcares (Diez de Baldeón, 1993: 299-310).

\section{El arte y las ciudades en el relato de Navagero.}

Se aprecia en todo el conjunto del relato de Navagero una preferencia por las obras de arte del gótico tardío, y un cierto desinterés y en ocasiones desdén por las primeras manifestaciones del Renacimiento en nuestro país. Esto puede parecer extraño de antemano, pero si lo analizamos con detenimiento es bastante lógico. A lo largo de su viaje por España recoge valoraciones de obras renacentistas. En su visita a Valladolid muestra más entusiasmo ante el colegio de San Gregorio, al que describe como fábrica suntuosa, de piedra labrada con ricos artesonados de oro (Navagero, 1983: 77), que ante el colegio de la Santa Cruz, en el que se introducía el lenguaje renacentista, de él solamente dice que también es un buen edificio.

Sabemos que la implantación del Renacimiento en España no fue homogénea, ni sigue las mismas fases que en Italia, sino que se va imponiendo poco a poco al dominante gótico final. Navagero venía de un país donde el arte renacentista ya era lo usual, y donde era puro y más perfecto que en ningún sitio, y quizás se quedara indiferente ante los tímidos balbuceos que se daban en España. Hagamos una comparación más para entender mejor el punto de vista de Navagero. Cuando visitó la Cartuja de Miraflores en Burgos se quedó fuertemente impresionado ante los sepulcros de Juan II e Isabel de Portugal, que le parecieron harto bellos (Navagero, 1983: 82) y, sin embargo, los sepulcros italianizantes de los Reyes Católicos de la Capilla Real de Granada eran harto bellos para España (Navagero, 1983: 73-74); como si no mereciesen ser considerados al lado de las obras renacentistas italianas.

Efectivamente podemos comprobar que Navagero se deja seducir por el lujo y la grandiosidad del gótico tardío antes que por las primeras manifestaciones renacentistas (Checa, 1992: 21-54), pero recordemos su opinión sobre el Hospital de Santa Cruz, que aunque no alude a la influencia italiana es bastante favorable. La cuestión es la razón por la que le gusta este edificio, si por su permanencia de elementos góticos o simplemente por la suntuosidad y profusión de su decoración aunque en ella se introduzcan elementos Rena- 
centistas. Muy posiblemente, los gustos estéticos de Navagero no se deban sólo al estilo, sino a la riqueza de los monumentos observados, cosa que no sería descabellada, porque en esta época, más que en ninguna otra, lo más apreciado era la riqueza de lo que se contempla y muestra de ello es la descripción del tesoro de la Catedral:

El tesoro de esta iglesia es además muy rico y está lleno de paños bordados de oro y de alhajas y preseas, dejadas por varios reyes y arzobispos para ornamento de la iglesia [...] Hay también una mitra muy rica con varias piedras muy buenas, $y$, aunque no tanto como dicen, vale mucho. Hay otras alhajas y perlas de que no hablo en particular, pero todas juntas son de gran valor, por lo que puede decirse que ésta es ciertamente la iglesia más rica de la cristiandad (Navagero, 1983: 112).

En el relato de Navagero las protagonistas son las ciudades, que son descritas con más detalle que los núcleos de población más pequeños. El autor apenas habla de algunos edificios de Talavera de la Reina y pasa totalmente por alto a Almagro, que en estos momentos era una localidad importante. Las ciudades de Castilla-La Mancha más presentes en el relato de Navagero son Toledo y Guadalajara. El embajador se detiene más en la primera que en la segunda, puesto que en la ciudad del Tajo permaneció más tiempo que en Guadalajara, que fue sólo una parada en el camino hacia la Corte.

El viajero también atiende a los aspectos sociales de los lugares visitados. En las dos ciudades más relevantes de Castilla-La Mancha Navagero percibió la importancia que tenía la nobleza, en Toledo por ser sede ocasional de la Corte y en Guadalajara atraídos por los Mendoza (García González, 2004: 120-121). En el caso de Toledo también da cuenta del poder que el clero tenía en la ciudad, que era la Sede Primada de la Iglesia española. Este poder irá en aumento en los años siguientes a la visita de Navagero y otros viajeros rememoraran sus palabras (Ruiz Gómez, 2009: 217-218). El relato del embajador veneciano es uno de los más importantes de su época, porque nos da una visión completa y ajustada a la realidad de las ciudades españolas y también de las de Castilla-La Mancha. 


\section{Bibliografía}

ARTEAGA, M.C. (1924): “La custodia de Arfe y sus predecesores en la catedral de Toledo". Boletín de la Sociedad Española de Excursiones, Sociedad Española de Excursiones, Madrid, págs. 238-254.

BAllesteros GAllardo, A. (1981): Patrimonio artístico de una ciudad: Talavera de la Reina. Ayuntamiento de Talavera de la Reina, Talavera de la Reina.

CARRERO DE DIOS, M. (1981): Las murallas y las puertas de Toledo. Instituto de Investigaciones y Estudios Toledanos, Toledo.

CHeCA CREMAdes, F. (1992): "Poder y piedad: patronos y mecenas en la introducción del Renacimiento en España”. Reyes y mecenas: Los Reyes Católicos, Maximiliano I y los inicios de la casa de Austria, Catálogo de la exposición, Ministerio de Cultura, Madrid, págs. 21-54.

COLOMBI-MONGUIÓ DE, A. (1992): “Boscán frente a Navagero: el nacimiento de la conciencia humanista en la poesía española". Nueva Revista de Filología Hispánica, Centro de Estudios Lingüísticos y Literarios, Méjico, pp. 143-168.

CONTARINI, T. (1666): Relation d'Hespagne Par monsieur Thom. Contarini Ambassadeur Ordinaire pour la S. République de Venize. Chez Claude Hyp, Montbeliard.

CORCHADO SORIANO, M. (1982): Los pueblos y sus términos. Instituto de Estudios Manchegos, Ciudad Real.

DieZ DE BALDEÓN, C. (1993): Almagro; arquitectura y sociedad. Junta de Comunidades de Castilla-La Mancha, Toledo.

DiEZ DEL CORRAL GARNICA, R. (1986): “La introducción del Renacimiento en Toledo: El hospital de Santa Cruz". Academia: Boletín de la Real Academia de Bellas Artes de San Fernando, Real Academia de Bellas Artes de San Fernando, Madrid, págs. 161-182.

FERnÁndez Collado, Á. (1999): La Catedral de Toledo en el siglo XVI. Diputación provincial, Toledo.

FERNÁNDEZ MADRID, M. T. (1991): El mecenazgo de los Mendoza en Guadalajara. Diputación Provincial e Instituto Provincial de Cultura, Guadalajara.

García GonZÁLeZ, F. (2004): Castilla-La Mancha en la Edad Moderna. Almud, Ciudad Real.

GONZÁLEZ MORENO, F. (1999-2000): “Una breve historia de la cerámica talaverana a través de los libros de viajes de extranjeros desde el siglo XII hasta el siglo XIX". Cuaderna: Revista de estudios humanísticos de Talavera y su antigua tierra, Colectivo Arrabal, Talavera de la Reina, págs. 221-238. 
LAYNA SERRANO, F. (1994): Historia de Guadalajara y sus Mendozas en los siglos XV y XVI. Aache, Guadalajara.

LAYNA SERRANO, F. (1997): El Palacio del Infantado en Guadalajara. Aache, Guadalajara.

LUPI, A. (1997): “Andrea Navagero o la poética de los Jardines: De los jardines humanísticos a los de España”. CRIADO Del VAL, M. (Dir.), Caminería Hispánica. Actas III congreso de Caminería Hispánica. Aache, Guadalajara, págs. 529-535.

MARIAS FRANCO, F. (1983): La arquitectura del Renacimiento en Toledo (1541-1631), v. I, Instituto Provincial de Investigaciones y Estudios Toledanos, Toledo.

MARIAS FRANCO, F. (1985): La arquitectura del Renacimiento en Toledo (1541-1631), v. II, Instituto Provincial de Investigaciones y Estudios Toledanos, Toledo.

Moreno Domínguez, L., Francisco J. A., PABlo A. (2002): El Toledo invisible. Antonio Pareja Editor, Toledo.

MüNZER, J. (2002): Viaje por España y Portugal. Polifemo, Madrid.

NAVAGERO, A. (1983): Viaje por España, Madrid (1524-1526). Antonio María Mabie ed., Madrid.

PERIS SÁNCHEZ, D. Coord. (1991): Arquitecturas de Toledo. Del Renacimiento al Racionalismo. Junta de Comunidades de Castilla-La Mancha, Toledo.

PORRES MARTÍN-ClETO, J. (1987): El artificio de Juanelo. Diputación Provincial, Toledo.

Rivero RodrígueZ, M. (2000): Diplomacia y relaciones exteriores en la Edad Moderna. De la cristiandad al sistema europeo, 1453-1794. Alianza, Madrid.

Romero MEdinA, R. (2010): “La casa del cardenal Mendoza en Guadalajara. Una traza del arquitecto Lorenzo Vázquez con la colaboración de canteros tardo góticos valencianos y maestros moros aragoneses". XII Encuentro de Historiadores del valle del Henares, separata, Institución de Estudios Complutenses, Institución Provincial de Cultura Marqués de Santillana, Centro de Estudios Seguntinos, Alcalá de Henares, págs. 1-21.

Ruibal, A. (1984): Calatrava la Vieja. Estudio de una fortaleza medieval. Instituto de Estudios Manchegos, Ciudad Real.

Ruíz Gómez, F. (2009): Castilla-La Mancha en su Historia. Junta de Comunidades de Castilla-La Mancha, Toledo.

SÁNCHEZ DONCEL, G. (1984): "El puente de Guadalajara sobre el Henares". Wad-al-Hayara. Instituto provincial de cultura Maques de Santillana, Guadalajara, págs. 227-238. 
SÁNCHEZ-PALENCIA, F. J., SAINZ PASCUAL, M. J. (1988): El circo romano de Toledo: estratigrafía y arquitectura. Concejalía de Educación y Cultura, Museo de Santa Cruz, Toledo.

SANTOYAla Heredero, L. (1990): “Las constituciones del Hospital de Santa Cruz (Toledo)". Espacio tiempo y forma serie IV Historia Moderna. Universidad de Educación a Distancia, Madrid, págs. 317-366.

ZALAMA RODRÍGUEZ, M. Á. (2005): "Isabel la Católica y las joyas. La custodia de la Catedral de Toledo". CHECA CREMADES, Fernando (coord.), El arte en la Corte de los Reyes Católicos. Rutas artísticas a principios de la Edad Moderna. Fundación Carlos de Amberes, Madrid, págs. 331-353. 